\title{
Majorana Fermion Surface Code for Universal Quantum Computation
}

\author{
Sagar Vijay, Timothy H. Hsieh, and Liang Fu \\ Department of Physics, Massachusetts Institute of Technology, Cambridge, Massachusetts 02139, USA
}

(Received 8 April 2015; published 10 December 2015)

\begin{abstract}
We introduce an exactly solvable model of interacting Majorana fermions realizing $Z_{2}$ topological order with a $Z_{2}$ fermion parity grading and lattice symmetries permuting the three fundamental anyon types. We propose a concrete physical realization by utilizing quantum phase slips in an array of Josephson-coupled mesoscopic topological superconductors, which can be implemented in a wide range of solid-state systems, including topological insulators, nanowires, or two-dimensional electron gases, proximitized by $s$-wave superconductors. Our model finds a natural application as a Majorana fermion surface code for universal quantum computation, with a single-step stabilizer measurement requiring no physical ancilla qubits, increased error tolerance, and simpler logical gates than a surface code with bosonic physical qubits. We thoroughly discuss protocols for stabilizer measurements, encoding and manipulating logical qubits, and gate implementations.
\end{abstract}

DOI: 10.1103/PhysRevX.5.041038

Subject Areas: Condensed Matter Physics,

Quantum Information,

Superconductivity

\section{INTRODUCTION}

As originally proposed by Ettore Majorana, the Majorana fermion is a particle that is its own antiparticle [1]. In the condensed-matter setting, Majorana fermions can emerge in topological superconductors as a special type of zero-energy quasiparticle that is bound to vortices or defects and formed by an equal superposition of electron and hole excitations $[2,3]$. Theory predicts that Majorana fermions can be created in a wide array of spinorbit-coupled materials in proximity to conventional superconductors [4-8]. Recent observations of zero-energy conductance peaks in such systems [9-12] provide encouraging hints of Majorana fermions [13,14].

Majorana fermions in topological superconductors are of great interest as they are predicted to produce exotic quantum phenomena such as the fractional Josephson effect [15-17] and electron teleportation [18]. Most remarkably, vortices or defects that carry Majorana fermions are predicted to exhibit non-Abelian statistics [19-21], which have yet to be observed in nature. In addition to its theoretical significance, non-Abelian statistics provides the foundation for topological quantum computation, in which logical qubits are encoded in the topologically degenerate states of non-Abelian anyons, and qubit operations are performed by braiding [22]. Topological quantum computation has the theoretical advantage of being immune to errors caused by local perturbations [23]. Demonstrating the non-Abelian

Published by the American Physical Society under the terms of the Creative Commons Attribution 3.0 License. Further distribution of this work must maintain attribution to the author(s) and the published article's title, journal citation, and DOI. statistics of Majorana fermions, however, requires braiding, fusing, and measuring the fusion outcome. This is a challenging task, as each of the above operations is yet to be experimentally achieved. Furthermore, braiding Majorana fermions alone is insufficient to perform the necessary gate operations for universal quantum computation.

The "surface code" [24,25] provides an alternative approach to universal quantum computation that uses measurements in an Abelian topological phase for gate operations and error correction. In the surface code, measurements of nontrivial commuting operators (stabilizers) are used to project onto a "code state," and logical qubits are effectively encoded in the anyon charge of a region by ceasing certain stabilizer measurements [26-29]. The logical gates necessary for universal quantum computation are realized through sequences of measurements used to move and braid the logical qubits. An advantage of the surface code architecture is its remarkable ability for error detection and subsequent correction during qubit readout, as the nucleation of anyons through the action of a random operator can be reliably tracked through stabilizer measurements. For a sufficiently low error rate per physical qubit measurement, scaling the size of the surface code produces an exponential suppression in propagated errors [30]. Remarkably, recent experiments with superconducting quantum circuits have demonstrated the ability to perform high-fidelity physical gate operations and reliable error correction for a surface code of small size [31-33].

In this work, we introduce a new scheme for surface code quantum computation that uses Majorana fermions as the fundamental physical degrees of freedom and exploits their unique properties for encoding and manipulating logical qubits. Our surface code is based on a novel $Z_{2}$ topological 
order with fermion parity grading (defined below), which we demonstrate in a class of exactly solvable Hamiltonians of interacting Majorana fermions, termed Majorana plaquette models. We demonstrate that charging energyinduced quantum phase slips in superconducting arrays with Majorana fermions generates the required multifermion plaquette interactions, providing a physical realization of our model. We then describe a detailed physical implementation of the "Majorana fermion surface code," including physical qubit and stabilizer measurements, the creation of logical qubits, error correction, and logical gate operations required for universal quantum computation.

The Majorana fermion surface code poses significant benefits over a surface code with bosonic physical qubits. First, stabilizer measurements in the Majorana surface code can be performed in a single step, whereas this requires several physical gate operations in the conventional surface code $[28,29]$. As a result, we anticipate that the Majorana surface code has a significantly higher error tolerance. Furthermore, our Majorana surface code operates with substantially less overhead, as it requires fewer physical qubits per encoded logical qubit and uses no physical ancilla qubits. Second, we may tune the energy gap for anyon excitations in our physical realization of the Majorana plaquette Hamiltonian, increasing error suppression in the Majorana fermion surface code. Finally, the lattice symmetries in the Majorana plaquette model permute the three fundamental anyon types, allowing for a much simpler implementation of the logical Hadamard gate. As we will show, these advantages arise from the unique approach taken by our Majorana fermion surface code and the use of Majorana fermions as the fundamental degrees of freedom. The fact that a Majorana fermion is "half" of an ordinary fermion-so that a pair is required to encode a qubit of information-is crucial to the Majorana fermion surface code. We emphasize that the non-Abelian statistics of Majorana-carrying vortices or defects is of no relevance to our proposal, as our code does not involve braiding them.

This paper is organized as follows. First, we introduce a solvable model of interacting Majorana fermions on the honeycomb lattice realizing a novel $Z_{2}$ topological order with a $Z_{2}$ fermion parity grading and an exact $S_{3}$ anyon symmetry. We propose a physical realization of this model that uses the charging energy in an array of mesoscopic superconductors [18] to implement the required nonlocal interactions between multiple Majorana fermions. Next, we demonstrate that our model provides a natural setting for the Majorana fermion surface code, in which a logical qubit is encoded in a set of physical qubits formed from Majorana fermions. We present a physical implementation of the Majorana surface code and propose detailed protocols for performing gate operations for universal quantum computation.

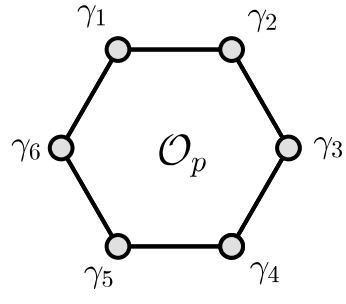

(a)

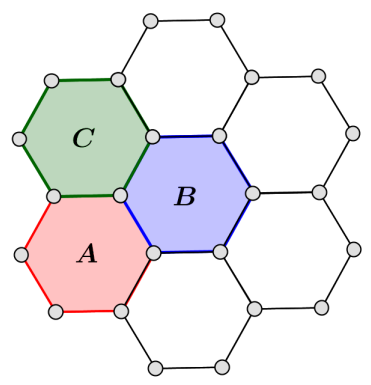

(b)
FIG. 1. We consider a honeycomb lattice with (a) a single Majorana fermion on each lattice site, so the $\mathcal{O}_{p}$ operator is the product of the six Majorana fermions on the vertices of a hexagonal plaquette. The colored plaquettes in (b) correspond to the three distinct bosonic excitations that may be obtained by violating a plaquette constraint.

\section{MAJORANA PLAQUETTE MODEL}

We begin by considering a honeycomb lattice with one Majorana fermion $(\gamma)$ on each lattice site; the Majorana fermions satisfy canonical anticommutation relations $\left\{\gamma_{n}, \gamma_{m}\right\}=2 \delta_{n m}$. The Hamiltonian is defined as the sum of operators acting on each hexagonal plaquette:

$$
H=-u \sum_{p} \mathcal{O}_{p}, \quad \mathcal{O}_{p} \equiv i \prod_{n \in \operatorname{vertex}(p)} \gamma_{n}
$$

We note that this model was mentioned in a work by Bravyi, Terhal, and Leemhuis [34], although its novel topological order and anyon excitations were not studied there; a closely related model in the same topological phase was introduced and studied by $\mathrm{Xu}$ and $\mathrm{Fu}$ [35].

It suffices to consider $u>0$ in the Hamiltonian (1), as the case with $u<0$ can be mapped to $u>0$ by changing the sign of the Majorana fermions on one sublattice. The operator $\mathcal{O}_{p}$ is the product of the six Majorana fermions on the vertices of plaquette $p$ as shown in Fig. 1(a). Since any two plaquettes on the honeycomb lattice share an even number of vertices, all of the plaquette operators commute, and the ground-state $\left|\Psi_{0}\right\rangle$ is defined by the condition

$$
\mathcal{O}_{p}\left|\Psi_{0}\right\rangle=\left|\Psi_{0}\right\rangle,
$$

for all plaquettes $p$. We note that, quite generally, Hamiltonians of interacting Majorana fermions with commuting terms may be realized on any lattice, so long as any pair of operators in the Hamiltonian only has overlapping support over an even number of Majorana fermions.

We demonstrate that the above Majorana plaquette model (1) realizes a $Z_{2}$ topological order of Fermi systems by considering the ground-state degeneracy and elementary excitations. First, we place the system on a torus by 
imposing periodic boundary conditions and find a four-fold degenerate ground state by counting the number of degrees of freedom and constraints on the full Hilbert space. For an $N$-site honeycomb lattice, the $2^{N / 2}$-dimensional Hilbert space of Majorana fermions is constrained by the fixed total fermion parity:

$$
\Gamma \equiv i^{N / 2} \prod_{n} \gamma_{n}
$$

For convenience, we choose a unit cell for the honeycomb lattice consisting of three plaquettes labeled $A, B$, and $C$, as shown in Fig. 1(b). We observe that on the torus, the product of plaquette operators on each of the $A-, B-$, and $C$-type plaquettes is equal to the total fermion parity:

$$
\Gamma=\prod_{p \in A} \mathcal{O}_{p}=\prod_{p \in B} \mathcal{O}_{p}=\prod_{p \in C} \mathcal{O}_{p} .
$$

The operators $\left\{\mathcal{O}_{p}\right\}$ on any one type of plaquette fix one-third of the plaquette eigenvalues via the condition (2) and impose $2^{N / 6-1}$ constraints on the Hilbert space. The number of unconstrained degrees of freedom is therefore given by

$$
D=2^{(N / 2)-1} /\left(2^{(N / 6)-1}\right)^{3}=4,
$$

which yields a four-fold ground-state degeneracy for the Majorana plaquette model on the torus.

The ground-state degeneracy is of a topological nature, as the four ground states are distinguished only by nonlocal operators. To see this, we construct a Wilson loop operator $W_{\ell}$, defined as a product of Majorana bilinears on a noncontractible loop $\ell$ on the torus:

$$
W_{\ell} \equiv \prod_{n, m \in \ell}\left(i \gamma_{n} \gamma_{m}\right)
$$

such that $W_{\ell}^{2}=1$ so that the Wilson loop has eigenvalues \pm 1 . Consider the operators $W_{x}$ and $W_{y}$ on the two nontrivial cycles of the torus $\ell_{x}$ and $\ell_{y}$, as shown in Fig. 2. Since $\ell_{x}$ and $\ell_{y}$ traverse an even number of vertices over any plaquette and do not contain any common lattice sites, we have $\left[W_{x}, W_{y}\right]=\left[W_{x}, H\right]=\left[W_{y}, H\right]=0$. Furthermore, we may construct Wilson loop operators $W_{\tilde{x}}$ and $W_{\tilde{y}}$ on loops $\tilde{\ell}_{x}$ and $\tilde{\ell}_{y}$, where $\tilde{\ell}_{x}$ is shifted from $\ell_{x}$ by a basis vector parallel to $\ell_{y}$ and likewise for $\tilde{\ell}_{y}$, such that $\left\{W_{\tilde{x}}, W_{y}\right\}=\left\{W_{\tilde{y}}, W_{x}\right\}=0$. As before, $W_{\tilde{x}}$ and $W_{\tilde{y}}$ commute with each other and with the Hamiltonian. Therefore, the four degenerate ground states may be distinguished by their eigenvalues under $W_{x}$ and $W_{y}$, with $W_{\tilde{x}}$ and $W_{\tilde{y}}$ transforming the ground states between distinct sectors. In analogy with conventional $Z_{2}$ gauge theory, we may identify the Wilson loop operators $W_{x, y}$ with electric

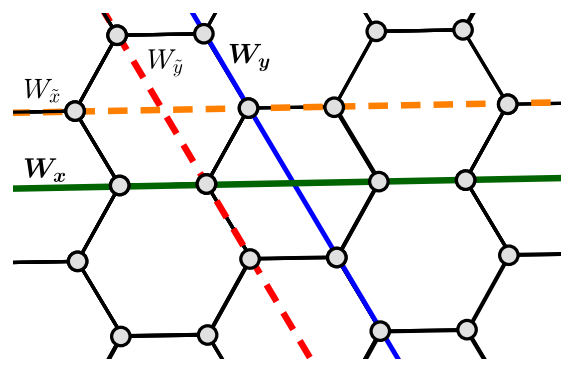

FIG. 2. The action of the commuting Wilson loop operators $W_{x}$ and $W_{y}$ is shown above as the product of the Majorana fermions on the lattice sites intersected by the appropriate colored lines. The operator $W_{\tilde{y}}$ anticommutes with $W_{x}$ and takes the ground state between two topological sectors.

charges traversing the torus in two different directions, and $W_{\tilde{x}, \tilde{y}}$ as magnetic fluxes on a dual lattice.

Gapped excitations above the ground state are obtained by flipping the eigenvalue of $\mathcal{O}_{p}$ from +1 to -1 on one or more plaquettes. Since the total fermion parity is fixed and equal to the product of all plaquette operators of each type, plaquette eigenvalues can only be flipped on pairs of plaquettes of the same type. This is achieved by string operators of the form (6), now acting on open paths and anticommuting with the plaquette operators at the two ends of the path, thereby creating a pair of anyon excitations.

An important feature of our Majorana plaquette model, the conservation of total fermion parity- a universal property of Fermi systems - makes it impossible to create or annihilate two excitations living on different types of plaquettes or to change one type of plaquette excitation into another. As a result, there are three distinct elementary plaquette excitations, labeled $A, B$, and $C$, by plaquette type. To determine their statistics, we braid these excitations by acting with Majorana hopping operators $i \gamma_{n} \gamma_{m}$ on lattice bonds [36]. We find that all three types of plaquette excitations have boson self-statistics and mutual semion statistics, i.e., braiding two distinct plaquette excitations generates a quantized Berry phase of $\pi$. From the elementary plaquette excitations, we may build composite excitations $A B, B C, A C$, and $A B C$ by flipping the eigenvalues of the $\mathcal{O}_{p}$ 's on two or three adjacent plaquettes. Among these, the composite excitation $A B C$ is simply a physical Majorana fermion since the Majorana operator $\gamma_{n}$ acting on a lattice site flips the eigenvalues of the $\mathcal{O}_{p}$ 's on the three surrounding $A, B$, and $C$ plaquettes. In contrast, the composite excitations $A B, B C$, and $A C$ are anyons, with fermion self-statistics and mutual semion statistics with the elementary excitations. We call these excitations composite Majorana fermions, as they are created by a string of physical Majorana fermions. A summary of the braiding statistics for all anyons in our Majorana plaquette model is given in the following table: 


\begin{tabular}{lcccccccc}
\hline & 1 & $A$ & $B$ & $C$ & $A B$ & $B C$ & $A C$ & $A B C$ \\
\hline 1 & +1 & +1 & +1 & +1 & +1 & +1 & +1 & +1 \\
$A$ & +1 & +1 & -1 & -1 & -1 & +1 & -1 & +1 \\
$B$ & +1 & -1 & +1 & -1 & -1 & -1 & +1 & +1 \\
$C$ & +1 & -1 & -1 & +1 & +1 & -1 & -1 & +1 \\
$A B$ & +1 & -1 & -1 & +1 & -1 & -1 & -1 & +1 \\
$B C$ & +1 & +1 & -1 & -1 & -1 & -1 & -1 & +1 \\
$A C$ & +1 & -1 & +1 & -1 & -1 & -1 & -1 & +1 \\
$A B C$ & +1 & +1 & +1 & +1 & +1 & +1 & +1 & -1 \\
\hline \hline
\end{tabular}

Strange as it may seem, the existence of eight types of quasiparticle excitations is a generic property of $Z_{2}$ topologically ordered phases in Fermi systems, due to the conservation of fermion parity. Consider artificially dividing the above quasiparticles into two groups: $(1, A, B, A B)$ and $(A B C, B C, A C, C)=A B C \times(1, A, B, A B)$. The former is equivalent to the four quasiparticles in $Z_{2}$ gauge theory coupled to a bosonic Ising matter field, as realized in Kitaev's toric code [22] or Wen's plaquette model [37]. The latter group of quasiparticles is obtained by attaching a physical Majorana fermion to the former. The conservation of total fermion parity guarantees that the two groups of quasiparticles cannot transform into each other in a closed system and thus have separate identities. We refer to the presence of two groups of excitations with different fermion parity as a $Z_{2}$ fermion parity grading.

A remarkable property of the Majorana plaquette model is that crystal symmetries of the honeycomb lattice permute the three fundamental anyon excitations, $A, B$, and $C$, by interchanging the three types of plaquettes. Examples of such lattice symmetries include $\pi / 3$ rotations about the center of a plaquette and translation by any primitive lattice vector. These symmetries of the honeycomb lattice provide a microscopic realization of the $S_{3}$ anyon symmetry that permutes quasiparticle sectors, as recently studied in the formalism of topological field theory by considering the symmetries of the $K$ matrices of Abelian topological states $[38,39]$.

\section{PHYSICAL REALIZATION}

\section{A. Physical platforms}

In this section, we show that the Majorana plaquette model can be physically realized in an array of mesoscopic topological superconductors that are Josephson coupled. A wide range of material platforms for engineering a topological superconductor have been proposed and are being experimentally studied [2,3]. As will be clear in the following, the scheme we propose for realizing the Majorana plaquette model is independent of which platform is used. For the sake of concreteness, we use a platform based on topological insulators in describing the general scheme below and discuss other platforms based on nanowires and a two-dimensional electron gas with spinorbit coupling in Sec. III C.

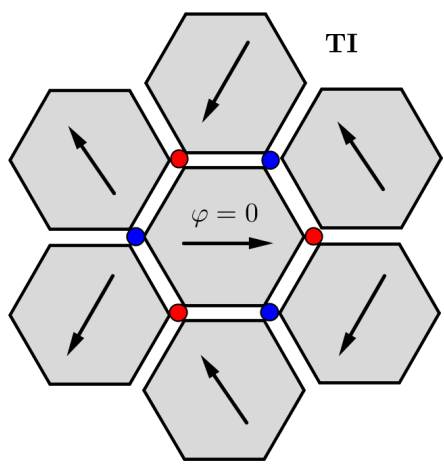

FIG. 3. Array of hexagonal $s$-wave superconducting islands placed on a TI surface. Each arrow points in the direction of the relative phase of the associated island, with $\varphi=0, \pm 2 \pi / 3$. This produces a honeycomb lattice of vortices (blue) and antivortices (red) at trijunctions, hosting Majorana fermions.

We place an array of hexagon-shaped $s$-wave superconducting islands on a topological insulator (TI) to induce a superconducting proximity effect on the TI surface states. The Hamiltonian for this superconductor-TI hybrid system is given by

$$
\begin{aligned}
H_{0}= & \int d \mathbf{r}(-i v) \psi^{\dagger}(\mathbf{r})\left(\partial_{x} s_{y}-\partial_{y} s_{x}-\mu\right) \psi(\mathbf{r}) \\
& +\sum_{j} \int d \mathbf{r}_{j}\left[\Delta e^{i \varphi_{j}} \psi_{\uparrow}^{\dagger}\left(\mathbf{r}_{j}\right) \psi_{\downarrow}^{\dagger}\left(\mathbf{r}_{j}\right)+\text { H.c. }\right],
\end{aligned}
$$

where $\psi=\left(\psi_{\uparrow}, \psi_{\downarrow}\right)^{T}$ is a two-component fermion field and $s_{x, y}$ are spin Pauli matrices. The first term describes the pristine TI surface states, with a single spin-nondegenerate Fermi surface and helical spin texture in momentum space. The second term describes the superconducting proximity effect: $\mathbf{r}_{j}$ belongs to the region underneath the $j$ th superconducting island, whose phase is denoted by $\varphi_{j}$.

As found by $\mathrm{Fu}$ and Kane [4], a vortex or antivortex trapped at a trijunction, where three islands meet, hosts a single Majorana fermion zero mode. Let us consider setting up the phases of superconducting islands to realize an array of vortices and antivortices at trijunctions. For example, the phases can be set to $\varphi_{j}=0,2 \pi / 3$, and $-2 \pi / 3$ on the $A-$, $B$-, and $C$-type islands, respectively, as shown in Fig. 3 . This yields a two-dimensional (2D) array of Majorana fermions on a honeycomb lattice. In practice, the desired phase configuration can be engineered by external electrical circuits [40] and/or magnetic flux. Alternatively, applying a perpendicular magnetic field generates a vortex lattice. These vortices may naturally sit at these trijunctions where the induced superconductivity is weak, leading to the desired lattice of Majorana fermions.

We take the size of the islands to be larger than the coherence length of the superconducting TI surface states. Under this condition, Majorana fermions at different sites have negligible wave-function overlap, preventing any 
unwanted direct coupling between them. (We note that even weak couplings from wave-function overlap will not affect the $Z_{2}$ topological order of the Majorana plaquette model because of its finite energy gap.) Nonetheless, as we show below, the charging energy of superconductors induces a nonlocal interaction between the six Majorana fermions on each island, providing the key ingredient of the Majorana plaquette model.

\section{B. Phase-slip-induced multifermion interactions}

The important but subtle interplay between Majorana fermions and charging energy was first recognized by $\mathrm{Fu}$ and formulated for superconductors with a fixed number of electrons [18]. Later works have extended this to multiple superconductors connected by Josephson coupling and single-electron tunneling [35,41-43]. In all of these cases, the charging energy of a given superconductor induces quantum phase slips $\varphi \rightarrow \varphi \pm 2 \pi$, from which the Majorana fermions in the superconductor acquire a minus sign: $\gamma_{i} \rightarrow-\gamma_{i}$. This property is due to the inherently double-valued dependence of Majorana operators on the superconducting phase [18].

In our setup for the Majorana plaquette model, the charging energy of the superconducting islands exerts even more dramatic and interesting effects on the Majorana fermions at trijunctions, which have not been previously studied. In the presence of a charging energy, the phase of each island becomes a quantum rotor. The kinetic energy of the rotor is provided by the charging energy $E_{c}$, which depends on the capacitance between an island and the rest of the array and is described by the following Hamiltonian:

$$
H_{c}=4 E_{c} \sum_{j}\left(\hat{n}_{j}-n_{g}\right)^{2},
$$

where $\hat{n}_{j} \equiv(-i) \partial / \partial \varphi_{j}$ is the Cooper-pair number operator for the $j$ th island and $n_{g}$ is the offset charge, which can be tuned by an externally applied electric field. The potential energy of the rotor is provided by the Josephson coupling $E_{J}$ between adjacent superconducting islands, given by

$$
H_{J}=-E_{J} \sum_{\left\langle j, j^{\prime}\right\rangle} \cos \left(\hat{\varphi}_{j}-\hat{\varphi}_{j^{\prime}}-a_{j j^{\prime}}\right),
$$

where $a_{j j^{\prime}}=\varphi_{0, j}-\varphi_{0, j^{\prime}}$ is externally set up such that the minimum of the Josephson energy corresponds to $\varphi_{j}=$ $\varphi_{0, j} \bmod 2 \pi$, with $\varphi_{0, j}=0,2 \pi / 3$, and $-2 \pi / 3$ for the $A, B$, and $C$-type islands, respectively.

Combining Eqs. (7), (8), and (9), the full Hamiltonian for our setup, i.e., an array of superconducting islands on a TI surface, is given by

$$
H=H_{0}+H_{c}+H_{J} .
$$

We work in the regime $E_{J} \gg E_{c}$. Under this condition, lowenergy states of the quantum rotor on a given island $\varphi_{j}$ consist of small-amplitude fluctuations around each potential minimum $\varphi_{0, j}+2 \pi m$. Moreover, different minima are connected by quantum phase slips, in which the phase $\varphi$ tunnels through a high-energy barrier to wind by $2 \pi n$, with $n$ an integer. The small-amplitude phase fluctuations around a potential minimum correspond to a quantum harmonic oscillator and thus generate a set of energy levels given by

$$
\epsilon_{\alpha}^{0} \approx(\alpha+1 / 2) \sqrt{8 E_{J} E_{c}},
$$

with $\alpha \in \mathbb{N}$.

On the other hand, quantum phase slips on a superconducting island strongly couple to the Majorana fermions that reside on the border with its neighbors, previously obtained by holding the phase fixed at $\varphi_{0, j}$. In other words, Majorana fermions enter the low-energy effective theory of Eq. (10) via quantum phase slips induced by the small charging energy on each superconducting island. This new physics makes our system different from a conventional Cooper-pair box. Remarkably, the action of a quantum phase slip involves Majorana fermions in a way that depends periodically on the phase winding number $n$ mod 6. Consider, for example, phase slips at the central superconducting island in Fig. 3. For $n=1$, a $2 \pi$ phase slip $\varphi=0 \rightarrow 2 \pi$ cyclically permutes the three Majorana fermions bound to vortices in the counterclockwise direction and the three Majorana fermions bound to antivortices in the clockwise direction, i.e.,

$$
\begin{array}{rrrr}
\varphi=0 \rightarrow 2 \pi: \gamma_{1} \rightarrow \gamma_{3}, & \gamma_{3} \rightarrow \gamma_{5}, & \gamma_{5} \rightarrow-\gamma_{1} \\
\gamma_{2} \rightarrow-\gamma_{6}, & \gamma_{4} \rightarrow \gamma_{2}, & \gamma_{6} \rightarrow \gamma_{4},
\end{array}
$$

as shown in Fig. 4, where $i=1, \ldots, 6$ labels the six Majorana fermions at vertices of this island in clockwise order. The physical movement of Majorana fermions induced by phase slips is a unique and attractive advantage of our setup, compared to other setups in which the positions of Majorana fermions are fixed [35,41-43]. On the other hand, for $n=3$, a $6 \pi$ phase slip takes each Majorana fermion over a full circle and back to its original position, from which it acquires a minus sign [4], i.e.,

$$
\varphi=0 \rightarrow 6 \pi: \gamma_{i} \rightarrow-\gamma_{i}
$$

Only for $n=6$ does each Majorana fermion come back to its original position unchanged.

We now add up the contributions of various phase slips to derive an effective Hamiltonian for Majorana fermions as a function of the offset charge $n_{g}$ for each state of the harmonic oscillator: 


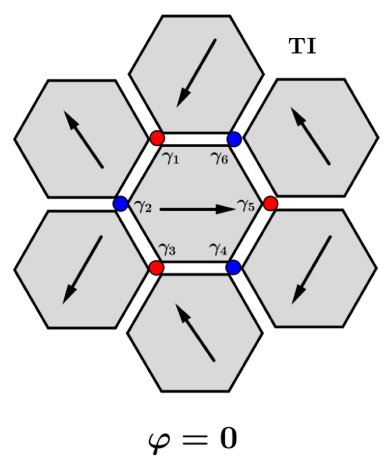

(a)

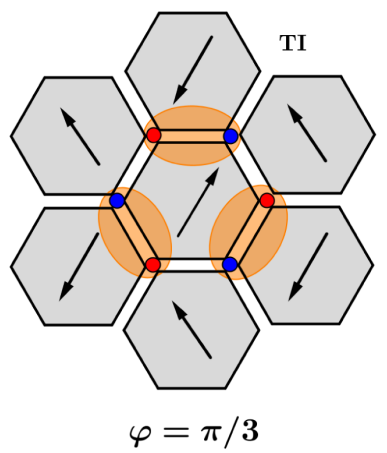

(b)

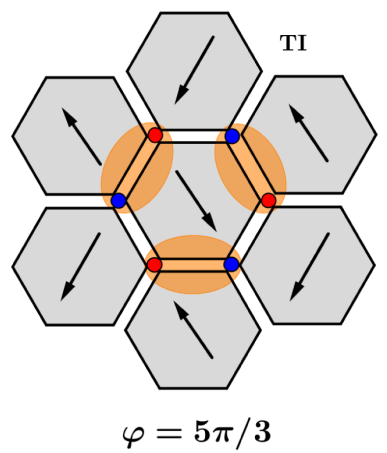

(c)

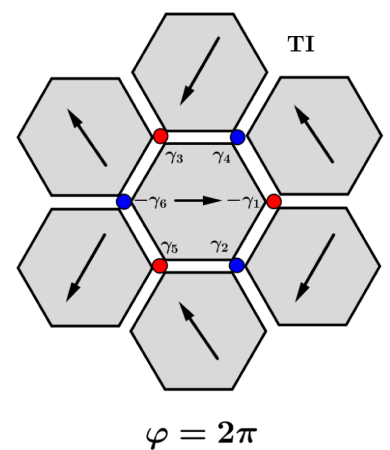

(d)

FIG. 4. Schematic of a $2 \pi$ phase slip on the central superconducting island in a hexagonal superconducting array on a TI surface, with the phase of the central island indicated in each panel. When the phase difference between neighboring islands is $\pi$, the pair of Majorana fermions on the shared edges couple [4] as indicated. The $2 \pi$ phase slip permutes the Majorana fermions as shown, leading to the transformation in Eq. (12).

$$
H_{\alpha}\left(n_{g}\right)=\epsilon_{\alpha}^{0}+\sum_{n=1}^{6}\left(t_{\alpha, n} \hat{U}_{n} e^{i 2 \pi n n_{g}}+\text { H.c. }\right) \text {. }
$$

Here, $\epsilon_{\alpha}^{0}$ is the quantized energy of the harmonic oscillator given by Eq. (11), which is the same for all internal states of the Majorana fermions. The second term describes quantum phase slips: $t_{\alpha, n}$ denotes the amplitude of the $\alpha$ th energy level of the harmonic oscillator tunneling between two potential minima that differ by $2 \pi n$, while $\hat{U}_{n}$ is the unitary operator acting on the Majorana fermions due to a $2 \pi n$ phase slip. The coupling $t_{\alpha, n}$ depends on the energy barrier in the phase-slip event and can be modulated by tuning $E_{c} / E_{J}$; for example, $t_{\alpha, 1} \propto e^{-\sqrt{8 E_{J} / E_{c}}}$ [44]. The offset charge $n_{g}$ provides an Aharonov-Bohm flux proportional to the winding number $n$.

The Hamiltonian (14) is analogous to the Bloch Hamiltonian that describes the band structure of a particle hopping in a one-dimensional periodic potential, with the offset charge $n_{g}$ playing the role of crystal momentum. Importantly, the phase particle carries internal degrees of freedom resulting from Majorana fermions $\gamma_{1}, \ldots, \gamma_{6}$ that are unique to our system. A phase slip that moves the phase particle to a different potential minimum also permutes the Majorana fermions as shown in Eqs. (12) and (13), similar to a spinful particle hopping in the presence of a nonAbelian gauge field. These permutations are represented by the unitary operators $\hat{U}_{n}$ in the effective Hamiltonian (14) acting on Majorana fermions. For example, the operator $\hat{U}_{1}$ that generates the transformation (12) is given by

$$
\begin{aligned}
\hat{U}_{1}= & \frac{1+\gamma_{2} \gamma_{3}}{\sqrt{2}} \frac{1+\gamma_{4} \gamma_{5}}{\sqrt{2}} \frac{1-\gamma_{6} \gamma_{1}}{\sqrt{2}} \\
& \times \frac{1+\gamma_{1} \gamma_{2}}{\sqrt{2}} \frac{1+\gamma_{3} \gamma_{4}}{\sqrt{2}} \frac{1+\gamma_{5} \gamma_{6}}{\sqrt{2}} .
\end{aligned}
$$

It follows from the addition of phase slips that $\hat{U}_{n}=\left(\hat{U}_{1}\right)^{n}$. In particular, the unitary operator $\hat{U}_{3}$, which takes $\gamma_{i}$ to $-\gamma_{i}$ as shown in Eq. (13), has a simple form

$$
\hat{U}_{3}=-\prod_{i=1}^{6} \gamma_{i}=i \mathcal{O}
$$

where $\mathcal{O}$ is the plaquette operator defined in the Majorana plaquette model (2). On the other hand, for $n=1,2,4$, or 5 , $U_{n}$ is a sum of operators $\gamma_{i} \gamma_{j}, \gamma_{i} \gamma_{j} \gamma_{k} \gamma_{l}$, and $i \mathcal{O}$.

Substituting the expressions for the $U_{n}$ 's into Eq. (14), we find that the effective Hamiltonian induced by the small charging energy of a single island takes the following form:

$$
H_{\alpha}\left(n_{g}\right)=\epsilon_{\alpha}^{0}+\Delta_{\alpha}\left(n_{g}\right) \mathcal{O}+V_{\alpha}\left(n_{g}\right),
$$

with

$$
\Delta_{\alpha}\left(n_{g}\right)=\sum_{m=1}^{5} t_{\alpha, m} \sin \left(2 \pi m n_{g}\right)
$$

$V_{\alpha}\left(n_{g}\right)$ includes a constant $t_{\alpha, 6} \cos \left(12 \pi n_{g}\right)$, as well as Majorana bilinear and quartic operators generated by phase slips with winding number $n \neq 0 \bmod 3$. Unlike $\mathcal{O}$, these operators on neighboring islands do not commute. However, by appropriately tuning $n_{g}$, the contribution of quartic operators to the effective Hamiltonian may vanish, so the only remaining terms in the Hamiltonian will be the six-Majorana interaction and Majorana bilinear terms. The bilinear term receives no contribution from any $\pm 6 \pi \mathrm{m}$ phase slip, while $\Delta_{\alpha}\left(n_{g}\right)$ receives contributions from every $\pm 2 \pi m$ phase-slip process. Therefore, for the remainder of this work, we assume that $V$ can be treated as a perturbation to the Majorana plaquette model that does not destroy the $Z_{2}$ topological order of the gapped phase. We note that an 
alternative setup without the presence of $V$ has been presented in a recent work [45].

\section{Discussion}

In deriving the effective Hamiltonian (14), we have implicitly assumed that Majorana fermions are the only low-lying excitations involved in phase-slip events, separated by an energy gap from other Andreev bound states in the junctions between islands. This assumption is valid because of the finite size of the islands, which leads to a discrete Andreev bound-state spectrum with a finite gap for all values of the phase. The presence of this gap justifies our derivation of the effective Hamiltonian (14) in a controlled manner.

Over the last few years, considerable experimental progress has been made in hybrid TI-superconducting systems. Proximity-induced superconductivity and supercurrents have been observed in a number of TI materials [46-52]. Low-temperature scanning tunneling microscopy (STM) experiments have found a proximity-induced superconducting gap on TI surface states, and the tunneling spectrum of Abrikosov vortices shows a zero-bias conductance peak, which is robust in a range of a magnetic field and splits at higher fields [12]. This peak has been attributed to the predicted Majorana fermion zero modes in the vortex cores of superconducting TI surface states. In view of these rapid, unabated advances, we regard the hybrid TI-superconductor system as a very promising material platform for realizing the Majorana plaquette model and studying the exciting physics of Majorana fermions enabled by quantum phase slips.

Besides TIs, a two-dimensional electron gas (2DEG) with spin-orbit coupling (such as InAs) can be driven into a helical state with an odd number of spin-polarized Fermi surfaces by an external Zeeman field, which provides another promising platform for realizing topological superconductivity via the proximity effect $[53,54]$. In this topological regime, vortices and trijunctions of a superconducting 2DEG host a single Majorana fermion, similar to the TI surface. Thus, our proposed setup for the Majorana plaquette model in Sec. III A directly applies to this system as well.

In addition to TIs and 2DEG, (quasi-)one-dimensional semiconductors and metals with strong spin-orbit coupling have become a hotly pursued system to search for Majorana fermions [5-7]. Signatures of Majorana fermions were reported in 2012, based on the observation of a zero-bias conductance peak in hybrid nanowire-superconductor systems $[9,10]$. One can envision a network of nanowires in proximity to Cooper-pair boxes to realize our Majorana plaquette model. In this direction, it is worth noting that a new physical system - a nanowire with an epitaxially grown superconductor layer-has recently been introduced to study Andreev bound states in the presence of charging energy [55].
Many other physical systems for Majorana fermions have been theoretically proposed and experimentally pursued; they are too numerous to list. Regardless of the particular system, nonlocal interactions between multiple Majorana fermions emerge from the charging energy of superconductors via quantum phase slips, and in the universal regime, such interactions are determined by the transformation of Majorana fermions under phase slips, as we have shown in Sec. III B.

Finally, we note several previous works related to our Majorana plaquette model and its physical realization. In Ref. [35], Xu and Fu first introduced a model of interacting Majorana fermions that realizes $Z_{2}$ topological order. This model involves four-body and eight-body plaquette interactions on square and octagonal plaquettes in a twodimensional lattice. Physical realizations of this model were proposed using an array of superconductor islands in proximity to either a 2D TI [35] or semiconductor nanowires $[56,57]$. The four-body nonlocal interaction between Majorana fermions comes directly from the charging energy, whereas the eight-body interaction comes from a high-order ring-exchange process generated by singleelectron tunneling between islands. In comparison, our Majorana plaquette model on the honeycomb lattice has the theoretical novelty of possessing an exact anyon permutation symmetry and can be realized in a much simpler manner using an array of superconductors on a 3D TI with global phase coherence, with all the required interactions coming directly from the charging energy. We also note a recent work on lattice models of Majorana fermions in Abrikosov vortices on a superconducting TI surface [58], which use different interactions and do not exhibit topological order.

\section{MAJORANA SURFACE CODE}

In the rest of this work, we demonstrate that the Majorana plaquette model finds a natural application as a Majorana fermion surface code, on which universal quantum computation and error correction may be performed. The main idea of the surface code is to (i) use anyons of the Majorana plaquette model to encode logical qubits, (ii) manipulate anyons to perform gate operations on logical qubits, and (iii) use commuting measurements of the Majorana plaquette operators for error correction. We describe the detailed implementation of the Majorana surface code, including the creation of logical qubits, error correction, and protocols for logical gate operations required for universal quantum computation.

The surface code architecture $[24,25,28]$ is a measurement-based scheme for quantum computation. It uses projective measurements of commuting operators-called "stabilizers"-acting on a 2D array of physical qubits to produce a highly entangled "code state" $|\psi\rangle$. Logical qubits are created by stopping the measurement of certain commuting operators to create "holes." The different possible 
anyon charges at a hole are the degrees of freedom that define a logical qubit. Logical gates are realized by manipulating and braiding holes via a sequence of measurements.

A key advantage of the surface code is its remarkable capability for error detection. The random measurement of an operator in the surface code corresponds to nucleating pairs of anyons, a process that can be reliably measured by tracking the eigenvalues of the commuting stabilizers. Reliable error detection hinges on having (i) a large number of physical qubits for a given encoded logical qubit and (ii) a sufficiently low error rate for stabilizer measurements [28]. For the previously studied surface code with bosonic physical qubits, it has been estimated $[59,60]$ that below a threshold as high as $1 \%$ error rate per physical qubit operation, scaling the size of the surface code permits an exponential suppression of errors propagated. This error tolerance makes the surface code architecture one of the most realistic approaches to practical, large-scale quantum computation.

Recent practical realizations of the surface code have used superconducting qubits coupled to a microwave transmission line resonator to perform qubit manipulations and measurements [31-33]. Here, a physical qubit is defined by two energy levels arising from quantization of phase fluctuations in a conventional Cooper-pair box. The surface code is implemented on a 2D array of physical qubits with the four-qubit interactions of Kitaev's toric code Hamiltonian [22] as the set of commuting stabilizers. The four-qubit stabilizer is measured by performing a sequence of single- and two-qubit gates between the four physical qubits and additional ancilla qubits [28]. Experiments have demonstrated the remarkable ability to operate these physical gates with fidelity above the threshold required for surface code error correction [31]. Recent experiments have also used error detection to preserve entangled code states on a surface code with a $9 \times 1$ [32] and a $2 \times 2$ [33] array of stabilizers. It remains to be shown that logical qubits can be successfully encoded and manipulated via logical gates in these surface code arrays.

\section{A. Implementation}

We implement the Majorana surface code on a 2D array of Majorana fermions by performing projective measurements of the Majorana plaquette operators $\left\{\mathcal{O}_{p}\right\}$, which form a complete set of commuting stabilizers. For the remainder of this paper, we will use "plaquette operators" and "stabilizers" interchangeably to refer to $\left\{\mathcal{O}_{p}\right\}$. A practical physical system for implementing the Majorana surface code is the superconductor-TI hybrid system introduced in the previous section. We place an array of superconducting islands on the TI surface, which are strongly Josephson coupled. By introducing external circuits or applying fluxes, we engineer the Josephson coupling between islands to achieve the phase

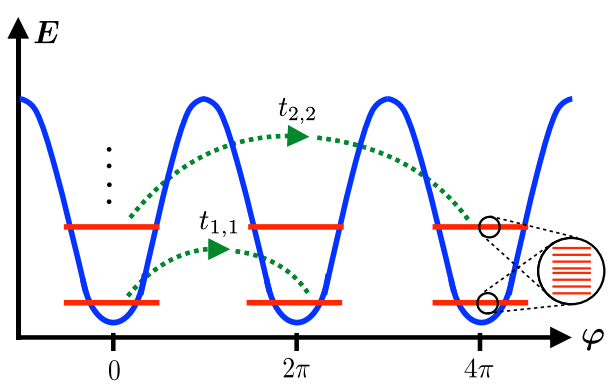

(a)

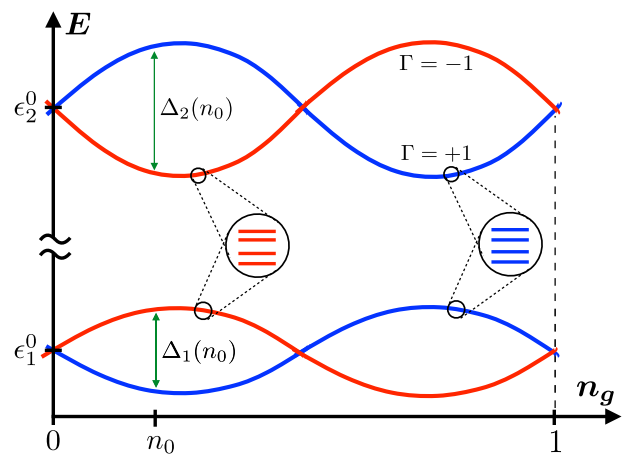

(b)

FIG. 5. (a) Schematic of the harmonic oscillator energy levels of the effective Hamiltonian (17), centered at $\varphi=2 \pi n$, with the $2 \pi$ and $4 \pi$ phase-slip amplitudes for the lowest energy levels shown. In (b), we show a schematic plot of the two lowest harmonic oscillator levels as a function of the gate charge. The energy splittings $\Delta_{1}$ and $\Delta_{2}$ are between states with even $(\Gamma=+1)$ and odd fermion parity $(\Gamma=-1)$ within the first and second harmonic oscillator levels, respectively. Each level within a fixed fermion parity sector is nearly four-fold degenerate.

configuration in Fig. 3, leading to a honeycomb lattice of Majorana fermions at trijunctions.

To perform a projective measurement of the Majorana plaquette operator on a given island, i.e., a single stabilizer, we decrease the Josephson coupling of the island with the rest of the array to activate quantum phase slips from the small but nonzero charging energy on this island. As shown by the effective Hamiltonian in Eq. (17), these quantum phase slips (partially) lift the degeneracy between states in the eight-dimensional Fock space of the six Majorana fermions. In particular, for every energy level of the harmonic oscillator, there is an energy splitting $\Delta_{\alpha}\left(n_{g}\right)$ between states of Majorana fermions with $\Gamma=+1$ (even fermion parity) and with $\Gamma=-1$ (odd fermion parity) from Eq. (18), where $\Gamma$ is the stabilizer eigenvalue; this is shown schematically in Fig. 5(b). Therefore, the charging energy of the island creates an energy difference between different stabilizer eigenstates. Furthermore, the energy gap between the two lowest harmonic oscillator levels on the island is a function of the stabilizer eigenvalue $\Gamma= \pm 1$, and in the limit of negligible interaction $V$, it takes the following form: 


$$
\Delta E_{\Gamma}\left(n_{g}\right)=\epsilon_{0}+\left[\Delta_{2}\left(n_{g}\right)-\Delta_{1}\left(n_{g}\right)\right] \Gamma+\cdots,
$$

where $\epsilon_{0} \equiv \epsilon_{2}^{0}-\epsilon_{1}^{0} \approx \sqrt{8 E_{J} E_{c}}$. The sensitivity of the energy gap to the stabilizer eigenvalue now permits a stabilizer measurement by simply measuring the energy gap. By shining a probe microwave beam on this island, we may measure the phase shift of the transmitted photons to determine the gap between the two harmonic oscillator levels $[61,62]$.

We now perform these stabilizer measurements on all of the superconducting islands to project onto an eigenstate of the Majorana plaquette Hamiltonian (1); this will be our reference "code state." We continue to perform measurements on all hexagonal islands in each cycle of the surface code in order to maintain the state. In subsequent cycles, we may encode logical qubits into the code state and manipulate the qubits via measurement. While projection onto the code state and error correction in the surface code rely exclusively on measuring the six-Majorana plaquette interaction, manipulation of logical qubits also requires measuring nearest-neighbor Majorana bilinears on the hexagonal lattice. This may be done by tuning the phase of neighboring superconducting islands to bring the pair of Majorana fermions on the shared edge sufficiently close together [4] so that the resulting wave-function overlap further splits the nearly four-fold degeneracy within a single fermion parity sector, shown in Fig. 5(b). Again, the Majorana bilinear may be measured by shining a probe beam to measure the energy gap to the next harmonic oscillator level.

Using the commuting six-Majorana operators in our plaquette model to realize a surface code provides unique advantages over the more conventional surface code with bosonic physical qubits. First, while a four-spin stabilizer measurement in the usual surface code requires performing 6-8 gates or measurements between a set of physical and ancilla qubits [28,59], stabilizer eigenvalues in the Majorana surface code are obtained via a single-step measurement by shining a probe beam. We emphasize that even when measurement is not being performed, the intrinsic charging energy of the islands generates a finite gap $\Delta_{1}\left(n_{g}\right)$ to creating anyon excitations and naturally suppresses errors at temperatures $k_{B} T<\Delta_{1}\left(n_{g}\right)$. We anticipate that the corresponding error tolerance for scalable quantum computation is substantially improved for the Majorana surface code. Second, the Majorana surface code operates with lower overhead than its bosonic counterpart, using three-qubit stabilizers and requiring no ancilla qubits. Finally, the anyon transmutation required to perform a logical Hadamard gate in the conventional surface code corresponds to a duality transformation that exchanges the star and plaquette toric code operators. This operation is quite difficult to perform on a single logical qubit as it also requires lattice surgery to patch the transformed logical qubit back into the remaining surface code $[28,63]$.
As lattice symmetries permute anyon sectors in the Majorana plaquette model, anyon transmutation in the Majorana surface code corresponds to a lattice translation of the logical qubit, substantially simplifying the Hadamard gate implementation.

\section{B. Logical qubits and error correction}

Logical qubits may be encoded in the surface code by ceasing the measurement of the plaquette operator on a hexagonal superconducting island in a surface code cycle, while continuing measurements on all other plaquettes. In theory, we could stop measuring a single plaquette and define a two-level system, with the $\hat{Z}$ and $\hat{X}$ operators of the logical qubit defined by the plaquette operator and a Wilson line connecting the plaquette to the boundary, respectively. A pair of such qubits on the $A$-type plaquettes is shown in Fig. 6(a), where the solid and dashed lines correspond to products of Majorana fermions that define the indicated logical operators. The two qubits shown may also be coherently manipulated by acting with the Wilson line operator connecting the two plaquettes, denoted $\hat{X}_{12}$.

In practice, however, it is difficult to manipulate qubits with an operator that connects to a distant boundary, so it is simpler to encode a logical qubit by stopping the stabilizer measurement on two well-separated plaquettes of the same type. We choose to only manipulate 2 of the 4 resulting degrees of freedom by defining $\hat{Z} \equiv \mathcal{O}_{p}$ and $\hat{X} \equiv W_{p q}$, the Wilson line operator connecting the two plaquettes. We use the opposite convention to define the logical $\hat{Z}$ and $\hat{X}$ operators for a qubit on the adjacent $B$ plaquettes; an example of such logical qubits is shown in Fig. 6(b). We note that when such a qubit is created, it is automatically initialized to an eigenstate of the plaquette operator, with the eigenvalue given by the measurement performed in the previous surface code cycle. As a result, logical qubits of type $A(B)$ are initialized to an eigenstate of the $\hat{Z}(\hat{X})$ logical operator.

To reduce errors during qubit manipulation, we may define a qubit by ceasing measurement of multiple adjacent plaquettes as shown in Fig. 6(b). In this particular case, the logical operator $\hat{X}$ is still a Wilson line connecting to another set of distant holes. However, the logical $Z$ is defined as $\hat{Z} \equiv \mathcal{O}_{p} \otimes \mathcal{O}_{q} \otimes \mathcal{O}_{r}$. For the remainder of our discussion, we consider logical qubits with only a single plaquette operator used to define the logical $\hat{Z}$; the generalization to larger qubits is straightforward.

Errors may occur during qubit manipulation, including (1) single-qubit errors due to the unintended measurement of a local operator involving an even number of Majorana fermions and (2) measurement errors. Single-qubit error correction may be performed on logical qubits by constantly measuring the remaining plaquette eigenvalues during surface code cycles. Since only pairs of plaquettes may be flipped simultaneously by a random measurement, corresponding to the nucleation of a pair of anyons of a 


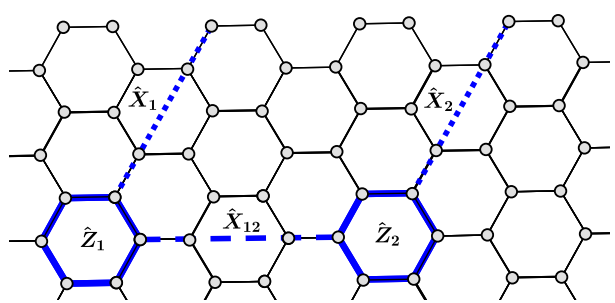

(a)

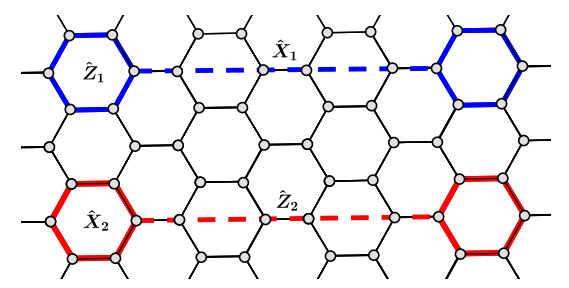

(b)

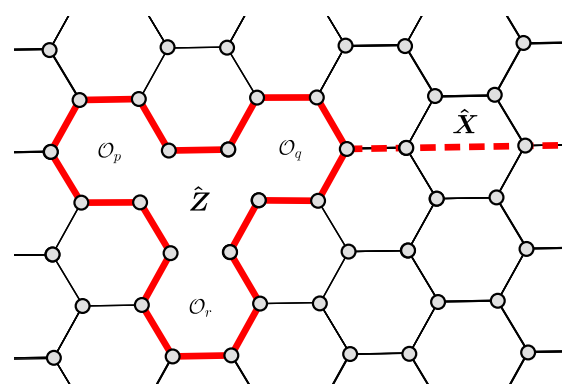

(c)

FIG. 6. Logical qubits in the Majorana surface code. In (a) we stop the measurement of two plaquette operators in subsequent surface code cycles, increasing the ground-state degeneracy by a factor of 4. If we take $\hat{Z}_{1}$ and $\hat{Z}_{2}$ to be the logical $\hat{Z}$ operators for the two encoded qubits, the corresponding $\hat{X}_{1}$ and $\hat{X}_{2}$ operators are given by Wilson lines connecting to the boundary. The two qubits may be coherently manipulated by applying the operator $\hat{X}_{12}$ as shown. In practice, it is simpler to define logical qubits by stopping the measurement of pairs of plaquettes of a single type, with the logical $\hat{X}$ and $\hat{Z}$ defined as shown in (b). We may also consider a logical qubit made of several "holes," as in (c), to minimize errors during qubit manipulation.

single type, detecting the change of an odd number of plaquette eigenvalues in a single surface code cycle will generally signal the presence of a random measurement performed on a nearby logical qubit. More precisely, when a stabilizer eigenvalue changes in a surface code cycle, it is efficient to store the location of that stabilizer and wait several code cycles, accumulating a spacetime diagram of stabilizer errors as additional errors occur $[30,59,60]$. After sufficiently many code cycles, the spacetime diagram may be used to determine the most likely configuration of Wilson lines that could have generated those errors $[28,29]$ using a minimum-weight perfect matching algorithm $[64,65]$. Errors may be subsequently corrected by software when performing logical qubit manipulations and readouts [28]. Random measurement errors involve incorrectly registering the eigenvalue of a plaquette operator; these are naturally corrected by performing multiple surface code cycles to verify the accuracy of a measurement.

\section{Logical gate implementations}

The Majorana surface code may be used for universal quantum computation by implementing CNOT, $T$, and Hadamard gates on logical qubits; this has been extensively studied in the context of the surface code architecture with underlying bosonic degrees of freedom $[28,63]$. Here, we describe the implementations of these gates in our realization of quantum computation with a Majorana surface code. Our gate implementations follow the spirit of the implementations presented in Ref. [28].

All gates in the Majorana surface code are implemented on logical qubits via a sequence of measurements. Let $\hat{U}$ be the desired unitary we wish to perform on the quantum state of several logical qubits, defined by the logical operators $\left\{\hat{X}_{i}\right\}$ and $\left\{\hat{Z}_{i}\right\}$. It is convenient to keep track of the transformation of the logical state by monitoring the transformation of logical operators $\hat{X}_{i} \rightarrow \hat{U} \hat{X}_{i} \hat{U}^{\dagger}$, $\hat{Z}_{i} \rightarrow \hat{U} \hat{Z}_{i} \hat{U}^{\dagger}$. In practice, performing the appropriate sequence of measurements will yield the transformation $W$, such that

$$
\begin{aligned}
& \hat{U} \hat{X}_{i} \hat{U}^{\dagger}= \pm \hat{W} \hat{X}_{i} \hat{W}^{\dagger}, \\
& \hat{U} \hat{Z}_{i} \hat{U}^{\dagger}= \pm \hat{W} \hat{Z}_{i} \hat{W}^{\dagger},
\end{aligned}
$$

where the signs depend on the outcomes of the specific measurements performed. These measurement outcomes are stored in software and used to correctly interpret the readout of a logical qubit.

In what follows, we often demonstrate our gate implementations in an "operator picture," where a set of operators in the surface code $\hat{o}_{1}, \ldots, \hat{o}_{n}$ and $\hat{p}_{1}, \ldots, \hat{p}_{m}$ with eigenvalues \pm 1 are measured in an appropriate sequence. This implements a logical gate via the desired transformations:

$$
\begin{aligned}
& \hat{Z} \rightarrow \hat{Z} \otimes \prod_{j=1}^{n} \hat{o}_{j}=\hat{U} \hat{Z} \hat{U}^{\dagger} \\
& \hat{X} \rightarrow \hat{X} \otimes \prod_{j=1}^{m} \hat{p}_{j}=\hat{U} \hat{X} \hat{U}^{\dagger}
\end{aligned}
$$

In practice, the measured outcomes for the $\left\{\hat{o}_{i}\right\}$ and $\left\{\hat{p}_{j}\right\}$ operators will be stored by software and used to obtain the above transformations during logical qubit readout.

CNOT gate.-A CNOT gate takes two qubits, a "control" and a "target," and flips the value of the target qubit based on the value of the control; it then returns the 
control unchanged. The action of a CNOT takes the following form in the basis of two-qubit states:

$$
\hat{C}=\left(\begin{array}{llll}
1 & 0 & 0 & 0 \\
0 & 1 & 0 & 0 \\
0 & 0 & 0 & 1 \\
0 & 0 & 1 & 0
\end{array}\right) .
$$

A CNOT gate may be implemented by braiding logical qubits in the Majorana surface code. In the simplest case, a CNOT between two logical qubits of different types is implemented through a single braiding operation that produces an overall sign if the hexagonal ends of both qubits contain an anyon, due to the $\pi$ mutual statistics, demonstrated in Sec. II. In the following section, we first describe the procedure to move a logical qubit along a given type of plaquette before discussing the braiding procedure required to produce a CNOT gate.

Consider the A-type logical qubit shown in Fig. 7. To move the qubit one unit to the right, we perform the following sequence of measurements. We begin by multiplying the $\hat{Z}$ logical operator by the eigenvalue of the adjacent $r$ plaquette operator to perform the transformation

$$
\hat{Z} \rightarrow \hat{Z}^{\prime} \equiv \hat{Z} \otimes \hat{\mathcal{O}}_{r}
$$

As the $r$ plaquette is being continuously measured, its eigenvalue $\mathcal{O}_{r}= \pm 1$ is known from the previous surface code cycle. In the next cycle, we stop measuring $\hat{\mathcal{O}}_{r}$ and measure the Majorana bilinear $i \gamma \eta$. We then multiply the $\hat{X}$ operator by the measurement outcome, affecting the transformation

$$
\hat{X} \rightarrow \hat{X}^{\prime} \equiv \hat{X} \otimes i \gamma \eta
$$

In the final surface code cycle, we begin measuring the original $\hat{Z}$ stabilizer and continue to include the measurement of the $\hat{Z}$ stabilizer in all subsequent surface code cycles. Furthermore, we redefine the logical operator $\hat{Z}^{\prime}$ as

$$
\hat{Z}^{\prime} \rightarrow \hat{Z}^{\prime \prime} \equiv \hat{Z}^{\prime} \otimes \hat{Z}
$$

The initial qubit configuration and final outcome are depicted schematically in Fig. 7. This sequence of measurements has shifted the $A$-type qubit by moving its hexagonal end one unit to the right, and it may generally be used to move an $A$ - or a $B$-type logical qubit within the $A$ or $B$ plaquettes, respectively.

We may now braid pairs of logical qubits to perform a CNOT gate in the Majorana surface code. The simplest CNOT that we may realize is between two distinct types of qubits, taking the $A$ qubit as the control, as shown in Fig. 8. Since the qubits are distinct, braiding the $B$-type qubitwith logical operators $\hat{X}_{B}$ and $\hat{Z}_{B}$-along a closed path $\ell$,

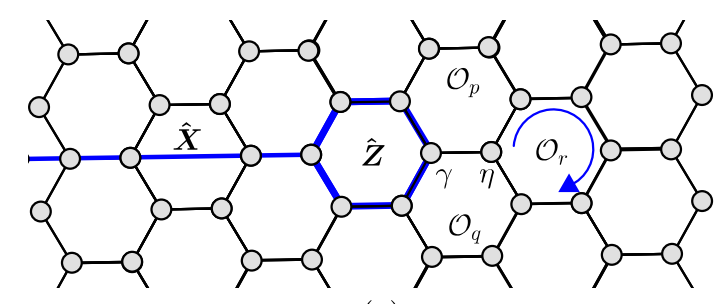

(a)

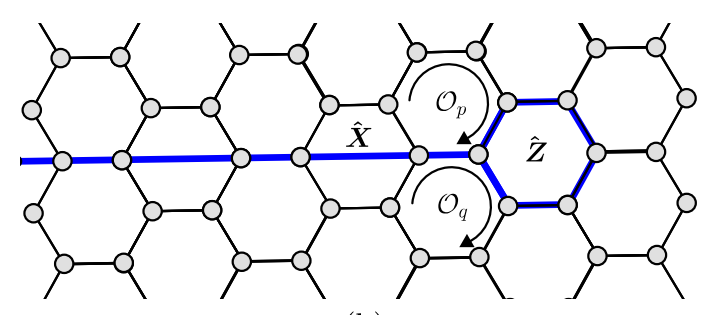

(b)

FIG. 7. We may move a logical qubit defined by $\hat{X}$ and $\hat{Z}$ operators along a given sublattice. We first multiply the logical $\hat{Z}$ by $\hat{\mathcal{O}}_{r}$. After measuring $i \gamma \eta$ in the next code cycle, we extend the $\operatorname{logical} \hat{X} \rightarrow \hat{X} \otimes i \gamma \eta$. Finally, we begin measuring $\hat{Z}$ in the next surface code cycle and restore $\hat{\mathcal{O}}_{p}$ and $\hat{\mathcal{O}}_{q}$ to six-Majorana operators.

enclosing the second qubit, (i) multiplies the Wilson line of the $B$-type qubit by the anyon charge enclosed by $\ell$ and (ii) multiplies the Wilson line of the $A$ qubit by the anyon charge of the $B$ qubit. This results in the transformation

$$
\hat{X}_{A} \rightarrow \hat{X}_{A} \otimes \hat{X}_{B}, \quad \hat{Z}_{B} \rightarrow \hat{Z}_{A} \otimes \hat{Z}_{B} \otimes \prod_{p \in \ell} \hat{\mathcal{O}}_{p},
$$

where $\left\{\hat{\mathcal{O}}_{p}\right\}$ are $A$ - and $C$-type plaquette operators enclosed by the braiding trajectory, as shown in Fig. 8 . Since the eigenvalues of the enclosed plaquette operators are known from the previous surface code cycle, we may implement the logical CNOT $\left(\hat{Z}_{A} \rightarrow \hat{Z}_{A}, \hat{Z}_{B} \rightarrow \hat{Z}_{A} \otimes \hat{Z}_{B}\right)$ by multiplying the transformed $\hat{Z}_{B}$ by an appropriate sign. In summary, the simplest braiding process between an $A$ and a $B$ logical qubit implements a CNOT on the $B$ qubit, with the $A$ qubit as the control.

A CNOT between two logical qubits of the same type may also be performed by appropriately braiding pairs of distinct types of logical qubits. In this case, we take one qubit as the control by convention and store the outcome of the CNOT gate in a third ancilla qubit. First, consider performing a CNOT gate on two $A$-type qubits. To implement the CNOT, we prepare two additional ancilla qubits; the first is an $A$ qubit prepared in the state $|\varphi\rangle \equiv$ $\left[\left|+_{z}\right\rangle+\left|-{ }_{z}\right\rangle\right] / \sqrt{2}$, while the second is a $B$ qubit prepared in the state $\left|+_{x}\right\rangle$, with $\left| \pm_{z}\right\rangle$ and $\left| \pm_{x}\right\rangle$ the eigenstates of the logical $Z$ and $X$ operators, respectively. Both ancilla qubits are prepared by measuring a +1 eigenvalue for the Wilson line joining the pair of plaquettes of the appropriate qubit. For the $A(B)$ qubit, this projects onto an eigenstate of the 


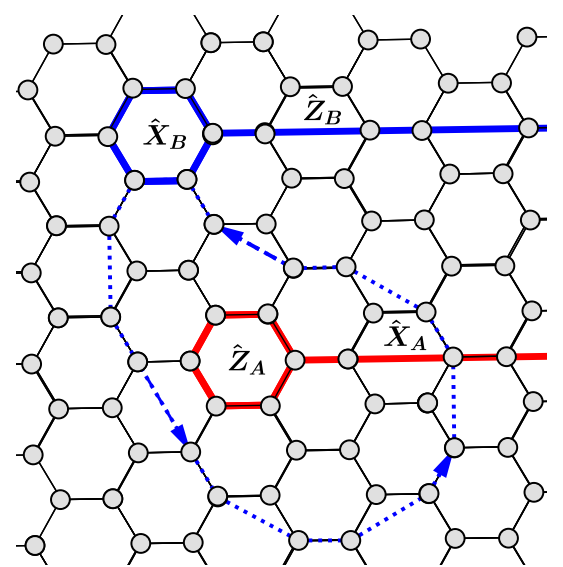

(a)

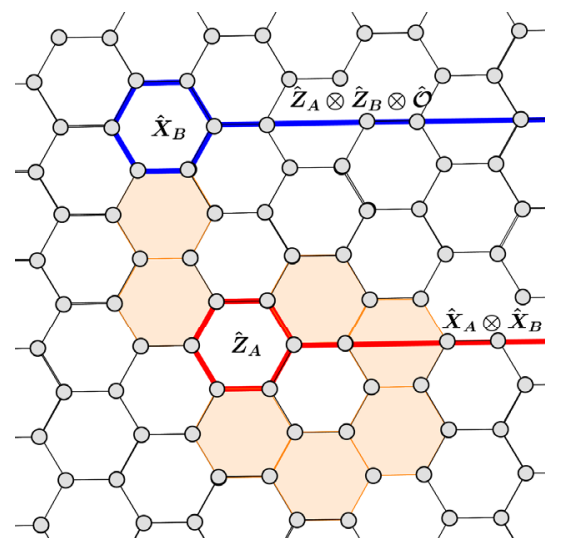

(b)

FIG. 8. CNOT gate. Braiding two logical qubits to perform a logical CNOT. In (a), a possible trajectory for braiding the first qubit around the second is indicated by the dotted line. Since the two qubits live on distinct sublattices, the braiding procedure induces the transformation $\hat{X}_{A} \rightarrow \hat{X}_{A} \otimes \hat{X}_{B}$ and $\hat{Z}_{B} \rightarrow$ $\hat{Z}_{A} \otimes \hat{Z}_{B} \otimes \hat{\mathcal{O}}$, where $\hat{\mathcal{O}}$ is the product of the colored plaquettes shown. As a result, this operation performs a CNOT transformation on the braided qubit.

logical $X(Z)$ operator and produces the desired ancilla states.

We now represent a complete basis of the four-qubit states as $\left|z_{B}, z_{c}, z_{t}, z_{\text {out }}\right\rangle$, referring to the eigenvalues of the logical $Z$ operators of the $B$ ancilla, the control, the target, and the ancilla $A$ qubits, respectively. We start out with an initial state $\left|\psi_{\text {init }}\right\rangle$ of the form

$$
\left|\psi_{\text {init }}\right\rangle \equiv \frac{1}{\sqrt{2}}\left[\left|+, z_{c}, z_{t},+\right\rangle+\left|+, z_{c}, z_{t},-\right\rangle\right]
$$

Next, we braid the $B$ ancilla qubit around all three remaining qubits as shown in Fig. 9(a). Up to an overall sign determined by the eigenvalues of plaquette operators enclosed by the braiding trajectory that are known from previous surface code cycles, this braiding implements the transformation $\hat{Z}_{B} \rightarrow \hat{Z}_{B} \otimes \hat{Z}_{c} \otimes \hat{Z}_{t} \otimes \hat{Z}_{\text {out }}$ on the logical

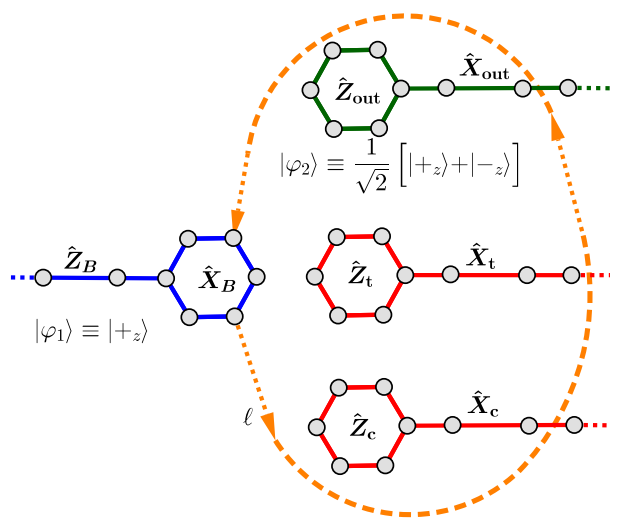

(a)

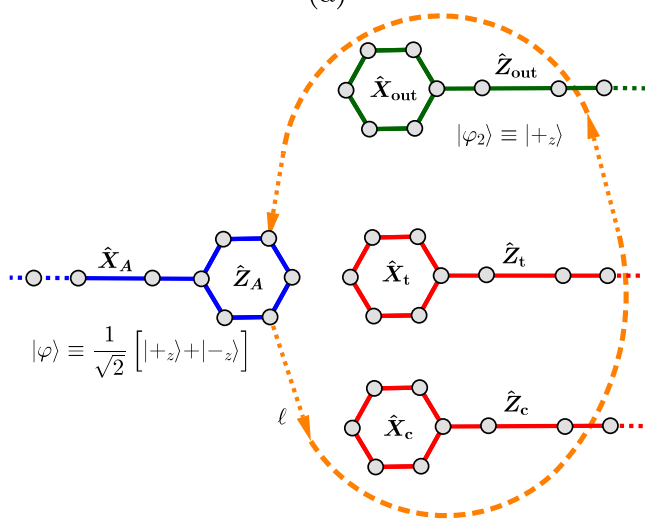

(b)

FIG. 9. Braiding processes that implement the transformation (a) $\hat{Z}_{a} \rightarrow \hat{Z}_{a} \otimes \hat{Z}_{c} \otimes \hat{Z}_{t} \otimes \hat{Z}_{\text {out }}$ up to an overall sign, as determined by the product of the appropriate plaquette operators enclosed by the path $\ell$, and (b) $\hat{Z}_{\text {out }} \rightarrow \hat{Z}_{\text {out }} \otimes \hat{Z}_{A}, \hat{Z}_{t} \rightarrow \hat{Z}_{t} \otimes \hat{Z}_{A}$, $\hat{Z}_{c} \rightarrow \hat{Z}_{c} \otimes \hat{Z}_{A}$. The two braids are used to realize CNOT gates between two (a) $A$-type and (b) $B$-type logical qubits, respectively. By convention, we take the lowest qubit enclosed by the braiding trajectory to be the control for the logical CNOT.

$Z$ of the ancilla qubit, where $\hat{Z}_{c}, \hat{Z}_{t}$, and $\hat{Z}_{\text {out }}$ are the logical $Z$ operators for the control, target, and ancilla $A$-type qubits, respectively. The final state we obtain is then of the form

$\left|\psi_{\text {final }}\right\rangle=\frac{1}{\sqrt{2}}\left[\left|z_{c} z_{t}, z_{c}, z_{t},+\right\rangle+\left|-z_{c} z_{t}, z_{c}, z_{t},-\right\rangle\right]$.

This braiding process is convenient, as a measurement of the state of the $B$ qubit can determine whether the state of the $A$ ancilla contains the correct outcome of the CNOT operation. If we now measure the logical $Z$ of the $B$ qubit and obtain $\hat{Z}_{B}=+1$, then we project onto a state with $z_{c} z_{t}=z_{\text {out }}$. In this case, the $A$ ancilla qubit contains the correct outcome of the CNOT between the other $A$ qubits. If $\hat{Z}_{B}=-1$, however, then $z_{c} z_{t}=-z_{\text {out }}$ and the $A$ ancilla contains the opposite of the correct CNOT outcome. In this case, we may act with $\hat{X}_{\text {out }}$ on the $A$ ancilla qubit in the surface code software [28] to obtain the desired final state. 
A similar process may be used to perform logical CNOT's between two $B$ qubits; now, we prepare an $A$ ancilla qubit and a $B$ ancilla qubit in the states shown in Fig. 9(b). After braiding the ancilla $A$ qubit around the control, target, and ancilla $B$ qubits, if we measure $\hat{X}_{A}=+1$, then the $B$ ancilla contains the desired outcome of the CNOT operation. Again, by convention, we take the control qubit to be the first one enclosed by the braiding trajectory, as shown in Fig. 9(b).

Hadamard gate.-The Hadamard gate is a single-qubit gate taking the matrix form

$$
\hat{H}=\frac{1}{\sqrt{2}}\left(\begin{array}{cc}
1 & 1 \\
1 & -1
\end{array}\right) .
$$

The action of a Hadamard gate is to exchange the logical $\hat{X}$ and $\hat{Z}$ operators so that $\hat{H} \hat{X} \hat{H}^{\dagger}=\hat{Z}$ and $\hat{H} \hat{Z} \hat{H}^{\dagger}=\hat{X}$. As the logical $\hat{X}$ and $\hat{Z}$ are defined oppositely on different types of qubits, a Hadamard operation in the bosonic surface code corresponds to an electric-magnetic duality transformation that interchanges star and plaquette operators in the toric code. In the ordinary surface code, such a transformation is quite difficult to implement, requiring a series of Hadamards on physical qubits enclosing the logical qubit so as to interchange the $\hat{X}$ and $\hat{Z}$ stabilizers, followed by physical swap gates in order to correctly patch the transformed logical qubit back into the remaining surface code array [63]. As lattice symmetries permute the anyons in the Majorana plaquette model, however, the logical Hadamard gate may be realized in the Majorana surface code by simply moving a logical qubit between distinct plaquettes.

We implement the logical Hadamard by the procedure shown in Fig. 10. Consider an $A$-type logical qubit. We multiply the logical $\hat{X}$ operator of the qubit, defined by the Wilson line in Fig. 10(a), by the product of the adjacent plaquette operators $\left\{\hat{\mathcal{O}}_{\mu_{k}}\right\}$ extending between the hexagonal ends of the qubit. The eigenvalues of these plaquette operators are known from previous surface code cycles. This operation implements the transformation

$$
\hat{X} \rightarrow \hat{Z}^{\prime} \equiv \hat{X} \otimes \prod_{k} \hat{\mathcal{O}}_{\mu_{k}} .
$$

At the same time, we multiply the logical $\hat{Z}$ by the adjacent plaquette operator $\hat{\mathcal{O}}_{p}$, shown in Fig. 10(a), that borders the logical qubit above:

$$
\hat{Z} \rightarrow \hat{X}^{\prime} \equiv \hat{Z} \otimes \hat{\mathcal{O}}_{p}
$$

In subsequent surface code cycles, we stop measuring the eigenvalue of $\hat{\mathcal{O}}_{p}$. We implement a similar transformation on the other hexagonal end of the logical qubit by stopping the measurement of the plaquette operator above the other qubit hole. The end result, after performing these

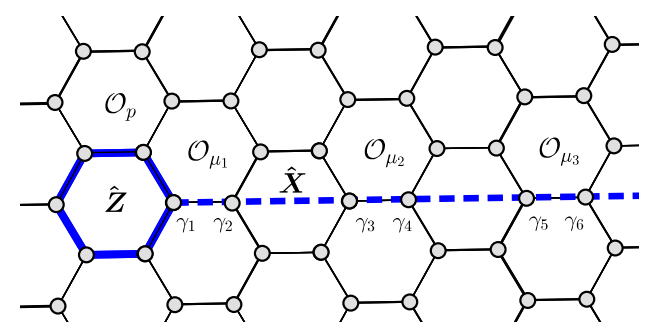

(a)

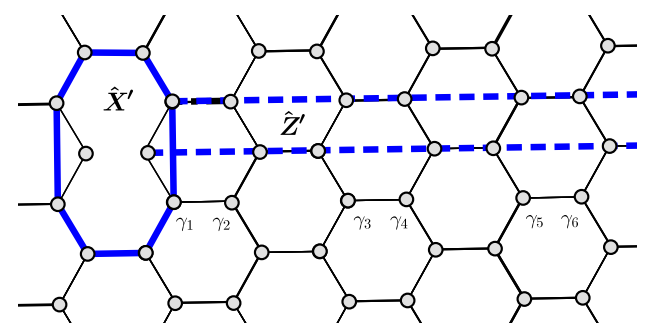

(b)

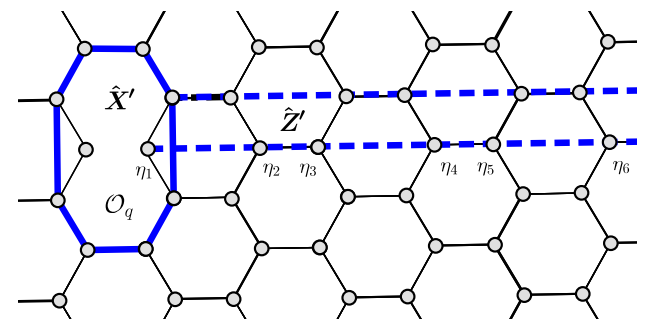

(c)

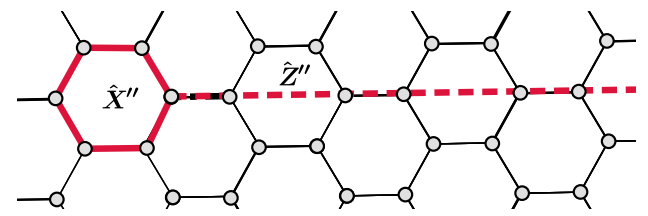

(d)

FIG. 10. Hadamard gate. A logical Hadamard is performed by transferring a qubit between distinct sublattices so that the logical $\hat{X}$ and $\hat{Z}$ operators are exchanged. We do this by taking the qubit in (a) and multiplying the logical $\hat{X}$ by the plaquette operators $\left\{\hat{\mathcal{O}}_{\mu_{k}}\right\}$ and the logical $\hat{Z}$ by $\hat{\mathcal{O}}_{p}$ and ceasing measurement of the fermion parity of plaquette $p$, yielding the operators shown in (b). Next, we measure the product $\left(i \eta_{1} \eta_{2}\right)\left(i \eta_{3} \eta_{4}\right) \ldots$ and $\hat{\mathcal{O}}_{q}$ and multiply by $\hat{Z}^{\prime}$ and $\hat{X}^{\prime}$, respectively. The final result is shown in (d).

operations, is shown in Fig. 10(b). The solid and dashed blue lines indicate the products of the Majorana fermions on the appropriate sites that define the $\hat{X}^{\prime}$ and $\hat{Z}^{\prime}$ operators, respectively.

In the next surface code cycle, we measure the product $\left(i \eta_{1} \eta_{2}\right)\left(i \eta_{3} \eta_{4}\right) \ldots$ of the Majorana fermions along the lower "string" that defines the $\hat{Z}^{\prime}$ operator; this measurement commutes with $\hat{X}^{\prime}$ since the two operators do not overlap, as shown in Fig. 10(c). Afterwards, we measure $\hat{\mathcal{O}}_{q}$, as well 
as the plaquette operator $\hat{\mathcal{O}}_{h}$ for the other hole of the original logical qubit. Then, we may perform the following transformations on the logical $\hat{X}^{\prime}$ and $\hat{Z}^{\prime}$ operators:

$$
\begin{aligned}
& \hat{X}^{\prime} \rightarrow \hat{X}^{\prime \prime} \equiv \hat{X}^{\prime} \otimes \hat{\mathcal{O}}_{q}, \\
& \hat{Z}^{\prime} \rightarrow \hat{Z}^{\prime \prime} \equiv \hat{Z}^{\prime} \otimes \prod_{\ell}\left(i \eta_{2 \ell-1} \eta_{2 \ell}\right) .
\end{aligned}
$$

This yields the logical qubit shown in Fig. 10(d). In subsequent surface code cycles, we continue measuring the eigenvalues of $\hat{\mathcal{O}}_{q}$ and $\hat{\mathcal{O}}_{h}$. Since the logical $\hat{Z}$ and $\hat{X}$ operators are defined differently on the $A$ and $B$-type plaquettes, our procedure for transforming our $A$ qubit into a $B$ qubit implements a logical Hadamard gate. An identical protocol may be used to perform a Hadamard gate on a logical $B$ qubit.

$S$ and $T$ gates.-Finally, we implement the logical $S$ and $T$ gates, described by the following single-qubit operations:

$$
\hat{S}=\left(\begin{array}{cc}
1 & 0 \\
0 & i
\end{array}\right), \quad \hat{T}=\left(\begin{array}{cc}
1 & 0 \\
0 & e^{i \pi / 4}
\end{array}\right) .
$$

As demonstrated in Ref. [28], it is possible to realize these gates by performing a series of logical Hadamard and CNOT gates between the logical qubit and an appropriate logical ancilla qubit. Here, we first discuss the $S$ - and $T$-gate implementations, given the appropriate ancilla qubit, before outlining a procedure for creating these logical ancillas in the surface code.

To implement an $S$ gate, we prepare a logical ancilla in the state

$$
\left|\varphi_{S}\right\rangle \equiv \frac{1}{\sqrt{2}}\left[\left|+_{z}\right\rangle+i\left|-{ }_{z}\right\rangle\right]
$$

Then, if $|\Psi\rangle$ is the state of the logical qubit of interest, the following sequence of logical Hadamard and CNOT gates implements the transformation $|\Psi\rangle \rightarrow \hat{S}|\Psi\rangle[28]$ :

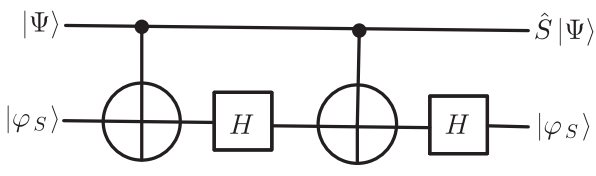

To perform a $T$ gate, we first prepare a logical ancilla in the state

$$
\left|\varphi_{T}\right\rangle \equiv \frac{1}{\sqrt{2}}\left[\left|+_{z}\right\rangle+e^{i \pi / 4}\left|-{ }_{z}\right\rangle\right]
$$

The $T$ gate is then implemented via a probabilistic circuit. We perform a CNOT between the ancilla and the logical qubit of interest and then measure the logical $\hat{Z}$ of the qubit.

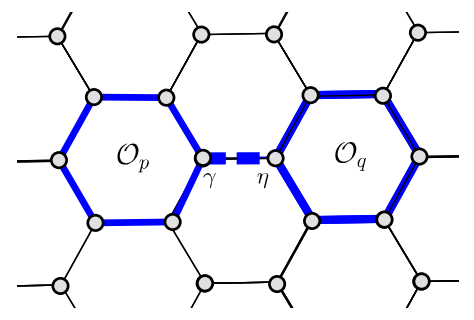

FIG. 11. $S$ - and T-gate ancilla preparation. We create the $\left|\varphi_{S}\right\rangle$ and $\left|\varphi_{T}\right\rangle$ ancilla states, which are needed to realize logical $S$ and $T$ gates by preparing the short qubit [28] shown above. We cease stabilizer measurements on two adjacent plaquettes $p$ and $q$. In the next surface code cycle, we perform a rotation of the two-level system defined by $i \gamma \eta$. Finally, we enlarge the logical qubit by extending one end of the qubit to guarantee stability against noise.

Depending on the measurement outcome, we implement an $S$ gate as shown below:

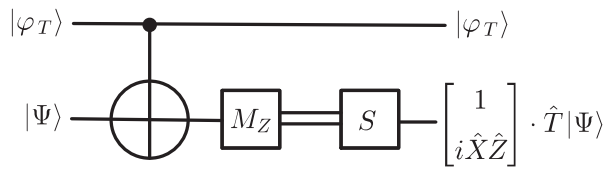

If the measurement outcome $M_{Z}=+1$, then we obtain the correct output $\hat{T}|\Psi\rangle$; otherwise, if $M_{Z}=-1$, then we have performed the transformation $|\Psi\rangle \rightarrow \hat{X} \hat{T}^{\dagger}|\Psi\rangle$. In this case, we implement an $S$ gate on the logical qubit and obtain the final state $i \hat{X} \hat{Z} \hat{T}|\Psi\rangle$. The action of the operator $i \hat{X} \hat{Z}$ may be undone in the surface code software to implement a pure $T$ gate on the logical qubit [28].

To realize the above implementations, we may prepare logical ancilla qubits in the states $\left|\varphi_{T}\right\rangle$ and $\left|\varphi_{S}\right\rangle$ as follows. First, we create a "short qubit" [28] by ceasing the fermion parity measurement on two adjacent plaquettes $p, q$ belonging to the same sublattice, as shown in Fig. 11(a). For this qubit, let $\hat{X} \equiv \hat{\mathcal{O}}_{p}$ and $\hat{Z} \equiv i \gamma \eta$. The qubit is initialized to a state $\left|\Psi_{ \pm}\right\rangle$such that $\hat{X}\left|\Psi_{ \pm}\right\rangle= \pm|\Psi\rangle$. In a basis of eigenstates of the logical $\hat{Z}$, the qubit state takes the form $\left|\Psi_{ \pm}\right\rangle=\left(\left|+_{z}\right\rangle \pm\left|-{ }_{z}\right\rangle\right) / \sqrt{2}$. Now, we assume that the two-level system formed by the pair of Majorana fermions $\gamma$ and $\eta$ can be manipulated by performing a rotation

$$
\hat{R}(\theta)=\left(\begin{array}{cc}
1 & 0 \\
0 & e^{i \theta}
\end{array}\right)
$$

that acts in the basis of $\left| \pm_{z}\right\rangle$ states. This may be implemented by using the phase of the adjacent superconducting islands to tune the coupling between the Majorana zero modes [66]. To prepare the state $\left|\varphi_{S}\right\rangle$, we perform the rotation $\hat{R}( \pm \pi / 2)\left|\Psi_{ \pm}\right\rangle$in the next surface code cycle, while to prepare $\left|\varphi_{T}\right\rangle$, we perform the operation 
$\hat{R}((2 \pi \pm \pi) / 4)\left|\Psi_{ \pm}\right\rangle$. Afterwards, to guarantee the stability of the qubit against noise generated by the environment, we increase the length of the logical $\hat{X}$ operator by extending one end of the logical qubit, as discussed in detail previously and shown schematically in Fig. 7. In practice, a high-fidelity implementation of the $S$ and $T$ gates requires that the short qubits are put through a distillation circuit, as discussed in Ref. [28], which may be implemented using a sequence of logical CNOT gates with other ancilla logical qubits.

We have presented a two-dimensional model of interacting Majorana fermions that realizes a novel type of $Z_{2}$ topological order with a microscopic $S_{3}$ anyon symmetry. The required multifermion interactions in the plaquette model are naturally generated by phase slips in an array of phase-locked $s$-wave superconducting islands on a TI surface. Based on this physical realization, we propose the Majorana surface code and provide the necessary measurement protocols and gate implementations for universal quantum computation. The Majorana surface code provides substantially increased error tolerance, reduced overhead, and simpler logical gate implementations over a surface code with bosonic physical qubits. We are optimistic that the Majorana fermion surface code will be physically implemented and may provide an advantageous platform for fault-tolerant quantum computation.

\section{ACKNOWLEDGMENTS}

We thank Patrick Lee for helpful comments and discussion. This work was supported by the Packard Foundation (L. F.), and the DOE Office of Basic Energy Sciences, Division of Materials Sciences and Engineering under Grant No. DE-SC0010526 (S. V. and T. H.). S. V. and L.F. conceived and developed the Majorana fermion surface code for universal quantum computation and wrote the manuscript. T. H. contributed to the analysis of the Majorana plaquette model.

[1] E. Majorana, Teoria Simmetrica dell'elettrone e del Positrone, Nuovo Cimento, 14, 171 (1937).

[2] J. Alicea, New Directions in the Pursuit of Majorana Fermions in Solid State Systems, Rep. Prog. Phys. 75, 076501 (2012).

[3] C. W. J. Beenakker, Search for Majorana Fermions in Superconductors, Annu. Rev. Condens. Matter Phys. 4, 113 (2013).

[4] L. Fu and C. L. Kane, Superconducting Proximity Effect and Majorana Fermions at the Surface of a Topological Insulator, Phys. Rev. Lett. 100, 096407 (2008).

[5] R. M. Lutchyn, J. D. Sau, and S. Das Sarma, Majorana Fermions and a Topological Phase Transition in Semiconductor-Superconductor Heterostructures, Phys. Rev. Lett. 105, 077001 (2010).
[6] Y. Oreg, G. Refael, and F. von Oppen, Helical Liquids and Majorana Bound States in Quantum Wires, Phys. Rev. Lett. 105, 177002 (2010).

[7] A. C. Potter and P. A. Lee, Multichannel Generalization of Kitaev's Majorana End States and a Practical Route to Realize Them in Thin Films, Phys. Rev. Lett. 105, 227003 (2010).

[8] S. Nadj-Perge, I. K. Drozdov, B. A. Bernevig, and A. Yazdani, Proposal for Realizing Majorana Fermions in Chains of Magnetic Atoms on a Superconductor, Phys. Rev. B 88, 020407(R) (2013).

[9] V. Mourik, K. Zuo, S. M. Frolov, S. R. Plissard, E. P. A. M. Bakkers, and L. P. Kouwenhoven, Signatures of Majorana Fermions in Hybrid Superconductor-Semiconductor Nanowire Devices, Science 336, 1003 (2012).

[10] A. Das, Y. Ronen, Y. Most, Y. Oreg, M. Heiblum, and H. Shtrikman, Zero-Bias Peaks and Splitting in an Al-InAs Nanowire Topological Superconductor as a Signature of Majorana Fermions, Nat. Phys. 8, 887 (2012).

[11] S. Nadj-Perge, I. K. Drozdov, J. Li, H. Chen, S. Jeon, J. Seo, A. H. MacDonald, B. Andrei Bernevig, and A. Yazdani, Observation of Majorana Fermions in Ferromagnetic Atomic Chains on a Superconductor, Science 346, 602 (2014).

[12] J.-P. Xu, M.-X. Wang, Z. L. Liu, J.-F. Ge, X. Yang, C. Liu, Z. A. Xu, D. Guan, C. L. Gao, D. Qian, Y. Liu, Q.-H. Wang, F.-C. Zhang, Q.-K. Xue, and J.-F. Jia, Experimental Detection of a Majorana Mode in the Core of a Magnetic Vortex inside a Topological Insulator-Superconductor $\mathrm{Bi}_{2} \mathrm{Te}_{3} / \mathrm{NbSe}_{2}$ Heterostructure, Phys. Rev. Lett. 114, 017001 (2015).

[13] K. T. Law, P. A. Lee, and T. K. Ng, Majorana Fermion Induced Resonant Andreev Reflection, Phys. Rev. Lett. 103, 237001 (2009).

[14] J. Liu, A. C. Potter, K. T. Law, and P. A. Lee, Zero-Bias Peaks in the Tunneling Conductance of Spin-Orbit-Coupled Superconducting Wires with and without Majorana End-States, Phys. Rev. Lett. 109, 267002 (2012).

[15] A. Kitaev, Unpaired Majorana Fermions in Quantum Wires, Phys. Usp. 44, 131 (2001).

[16] H.-J. Kwon, K. Sengupta, and V. M. Yakovenko, Fractional ac Josephson Effect in p- and d-wave Superconductors, Eur. Phys. J. B 37, 349 (2003).

[17] L. Fu and C. L. Kane, Josephson Current and Noise at a Superconductor-Quantum Spin Hall InsulatorSuperconductor Junction, Phys. Rev. B 79, 161408(R) (2009).

[18] L. Fu, Electron Teleportation via Majorana Bound States in a Mesoscopic Superconductor, Phys. Rev. Lett. 104, 056402 (2010).

[19] G. Moore and N. Read, Nonabelions in the Fractional Quantum Hall Effect, Nucl. Phys. B360, 362 (1991).

[20] N. Read and D. Green, Paired States of Fermions in Two Dimensions with Breaking of Parity and Time-Reversal Symmetries and the Fractional Quantum Hall Effect, Phys. Rev. B 61, 10267 (2000).

[21] D. A. Ivanov, Non-Abelian Statistics of Half-Quantum Vortices in p-Wave Superconductors, Phys. Rev. Lett. 86, 268 (2001).

[22] A. Kitaev, Fault-Tolerant Quantum Computation by Anyons, Ann. Phys. (Amsterdam) 303, 2 (2003). 
[23] C. Nayak, S. H. Simon, A. Stern, M. Freedman, and S. Das Sarma, Non-Abelian Anyons and Topological Quantum Computation, Rev. Mod. Phys. 80, 1083 (2008).

[24] S. Bravyi and A. Kitaev, Quantum Codes on a Lattice with Boundary, Quantum Computers and Computing 2, 43 (2001).

[25] M. H. Freedman and D. A. Meyer, Projective Plane and Planar Quantum Codes, Found. Comput. Math. 1, 325 (2001).

[26] R. Raussendorf and J. Harrington, Fault-Tolerant Quantum Computation with High Threshold in Two Dimensions, Phys. Rev. Lett. 98, 190504 (2007).

[27] R. Raussendorf, J. Harrington, and K. Goyal, Topological Fault-Tolerance in Cluster State Quantum Computation, New J. Phys. 9, 199 (2007).

[28] A. G. Fowler, M. Mariantoni, J. M. Martinis, and A. N. Cleland, Surface Codes: Towards Practical Large-Scale Quantum Computation, Phys. Rev. A 86, 032324 (2012).

[29] A. G. Fowler, A. M. Stephens, and P. Groszkowski, HighThreshold Universal Quantum Computation on the Surface Code, Phys. Rev. A 80, 052312 (2009).

[30] E. Dennis, A. Kitaev, A. Landahl, and J. Preskill, Topological Quantum Memory, J. Math. Phys. (N.Y.) 43, 4452 (2002).

[31] R. Barends, J. Kelly, A. Megrant, A. Veitia, D. Sank, E. Jeffrey, T. White, J. Mutus, A. Fowler, B. Campbell et al., Superconducting Quantum Circuits at the Surface Code Threshold for Fault Tolerance, Nature (London) 508, 500 (2014).

[32] J. Kelly, R. Barends, A. Fowler, A. Megrant, E. Jeffrey, T. White, D. Sank, J. Mutus, B. Campbell, Y. Chen et al., State Preservation by Repetitive Error Detection in a Superconducting Quantum Circuit, Nature (London) 519, 66 (2015).

[33] A. D. Córcoles, E. Magesan, S. J. Srinivasan, A. W. Cross, M. Steffen, J. M. Gambetta, and J. M. Chow, Demonstration of a Quantum Error Detection Code Using a Square Lattice of Four Superconducting Qubits, Nat. Commun. 6, 6979 (2015).

[34] S. Bravyi, B. Terhal, and B. Leemhuis, Majorana Fermion Codes, New J. Phys. 12, 083039 (2010).

[35] C. Xu and L. Fu, Fractionalization in Josephson Junction Arrays Hinged by Quantum Spin Hall Edges, Phys. Rev. B 81, 134435 (2010).

[36] See Supplemental Material at http://link.aps.org/ supplemental/10.1103/PhysRevX.5.041038 for a derivation of the braiding and self-statistics of the topological excitations in the Majorana plaquette model and a discussion of how lattice symmetries in the model provide a microscopic realization of the $S_{3}$ anyon symmetry.

[37] X.-G. Wen, Quantum Orders in an Exact Soluble Model, Phys. Rev. Lett. 90, 016803 (2003).

[38] A. Kitaev, Anyons in an Exactly Solved Model and Beyond, Ann. Phys. (Amsterdam) 321, 2 (2006).

[39] J. C. Y. Teo, A. Roy, and X. Chen, Unconventional Fusion and Braiding of Topological Defects in a Lattice Model, Phys. Rev. B 90, 115118 (2014).

[40] D. Van Harlingen, private communication.
[41] B. van Heck, F. Hassler, A. R. Akhmerov, and C. W. J. Beenakker, Coulomb Stability of the $4 \pi$-Periodic Josephson Effect of Majorana Fermions, Phys. Rev. B 84, 180502(R) (2011).

[42] R. Hutzen, A. Zazunov, B. Braunecker, A. L. Yeyati, and R. Egger, Majorana Single-Charge Transistor, Phys. Rev. Lett. 109, 166403 (2012).

[43] B. van Heck, A. R. Akhmerov, F. Hassler, M. Burrello, and C. W. J. Beenakker, Coulomb-Assisted Braiding of Majorana Fermions in a Josephson Junction Array, New J. Phys. 14, 035019 (2012).

[44] J. Koch, T. M. Yu, J. Gambetta, A. A. Houck, D. I. Schuster, J. Majer, A. Blais, M. H. Devoret, S. M. Girvin, and R. J. Schoelkopf, Charge-Insensitive Qubit Design Derived from the Cooper Pair Box, Phys. Rev. A 76, 042319 (2007).

[45] S. Vijay and L. Fu, Physical Implementation of a Majorana Fermion Surface Code for Fault-Tolerant Quantum Computation, arXiv:1509.08134.

[46] M. Veldhorst, M. Snelder, M. Hoek, T. Gang, V. K. Guduru, X. L. Wang, U. Zeitler, W. G. van der Wiel, A. A. Golubov, H. Hilgenkamp, and A. Brinkman, Josephson Supercurrent through a Topological Insulator Surface State, Nat. Mater. 11, 417 (2012).

[47] J. R. Williams, A. J. Bestwick, P. Gallagher, S. S. Hong, Y. Cui, A. S. Bleich, J. G. Analytis, I. R. Fisher, and D. Goldhaber-Gordon, Unconventional Josephson Effect in Hybrid Superconductor-Topological Insulator Devices, Phys. Rev. Lett. 109, 056803 (2012).

[48] F. Yang, F. Qu, J. Shen, Y. Ding, J. Chen, Z. Ji, G. Liu, J. Fan, C. Yang, L. Fu, and L. Lu, Proximity-Effect-Induced Superconducting Phase in the Topological Insulator $\mathrm{Bi}_{2} \mathrm{Se}_{3}$, Phys. Rev. B 86, 134504 (2012).

[49] J. B. Oostinga, L. Maier, P. Schuffelgen, D. Knott, C. Ames, C. Brune, G. Tkachov, H. Buhmann, and L. W. Molenkamp, Josephson Supercurrent through the Topological Surface States of Strained Bulk HgTe, Phys. Rev. X 3, 021007 (2013).

[50] I. Sochnikov, A. J. Bestwick, J. R. Williams, T. M. Lippman, I. R. Fisher, D. Goldhaber-Gordon, J. R. Kirtley, and K. A. Moler, Direct Measurement of Current-Phase Relations in Superconductor/Topological Insulator/Superconductor Junctions, Nano Lett. 13, 3086 (2013).

[51] S. Cho, B. Dellabetta, A. Yang, J. Schneeloch, Z. Xu, T. Valla, G. Gu, M.J. Gilbert, and N. Mason, Symmetry Protected Josephson Supercurrents in Three-Dimensional Topological Insulators, Nat. Commun. 4, 1689 (2013).

[52] C. Kurter, A. D. K. Finck, P. Ghaemi, Y. S. Hor, and D. J. Van Harlingen, Dynamical Gate-Tunable Supercurrents in Topological Josephson Junctions, Phys. Rev. B 90, 014501 (2014).

[53] J. D. Sau, R. M. Lutchyn, S. Tewari, and S. Das Sarma, Generic New Platform for Topological Quantum Computation Using Semiconductor Heterostructures, Phys. Rev. Lett. 104, 040502 (2010).

[54] J. Alicea, Majorana Fermions in a Tunable Semiconductor Device, Phys. Rev. B 81, 125318 (2010).

[55] A. P. Higginbotham, S. M. Albrecht, G. Kirsanskas, W. Chang, F. Kuemmeth, P. Krogstrup, T. S. Jespersen, J. Nygard, K. Flensberg, and C. M. Marcus, Parity Lifetime 
of Bound States in a Proximitized Semiconductor Nanowire, arXiv:1501.05155.

[56] B. M. Terhal, F. Hassler, and D. P. DiVincenzo, From Majorana Fermions to Topological Order, Phys. Rev. Lett. 108, 260504 (2012).

[57] Z. Nussinov, G. Ortiz, and E. Cobanera, Arbitrary Dimensional Majorana Dualities and Architectures for Topological Matter, Phys. Rev. B 86, 085415 (2012).

[58] C. K. Chiu, D. I. Pikulin, and M. Franz, Strongly Interacting Majorana Fermions, Phys. Rev. B 91, 165402 (2015).

[59] D. S. Wang, A. G. Fowler, and L. C. L. Hollenberg, Surface Code Quantum Computing with Error Rates over 1\%, Phys. Rev. A 83, 020302(R) (2011).

[60] D. S. Wang, A. G. Fowler, A. M. Stephens, and L. C. L. Hollenberg, Threshold Error Rates for the Toric and Surface Codes, Quantum Inf. Comput. 10, 456 (2010).

[61] F. Hassler, A. R. Akhmerov, and C. W. J. Beenakker, The Top-Transmon: A Hybrid Superconducting Qubit for
Parity-Protected Quantum Computation, New J. Phys. 13, 095004 (2011).

[62] L. Sun, L. DiCarlo, M. D. Reed, G. Catelani, Lev S. Bishop, D. I. Schuster, B. R. Johnson, G. A. Yang, L. Frunzio, L. Glazman, M. H. Devoret, and R. J. Schoelkopf, Measurements of Quasiparticle Tunneling Dynamics in a Band-GapEngineered Transmon Qubit, Phys. Rev. Lett. 108, 230509 (2012).

[63] A. G. Fowler, Low-Overhead Surface Code Logical Hadamard, Quantum Information Computation 12, 970 (2012).

[64] J. Edmonds, Paths, Trees, and Flowers, Can. J. Math. 17, 449 (1965).

[65] A. G. Fowler, A. C. Whiteside, and L. C. L. Hollenberg, Towards Practical Classical Processing for the Surface Code, Phys. Rev. Lett. 108, 180501 (2012).

[66] L. Jiang, C. L. Kane, and J. Preskill, Interface between Topological and Superconducting Qubits, Phys. Rev. Lett. 106, 130504 (2011). 